\title{
RECOGNIZING THE TOPOLOGY OF THE SPACE OF CLOSED CONVEX SUBSETS OF A BANACH SPACE
}

\author{
TARAS BANAKH, IVAN HETMAN, AND KATSURO SAKAI
}

\begin{abstract}
Let $X$ be a Banach space and $\operatorname{Conv}_{\mathrm{H}}(X)$ be the space of non-empty closed convex subsets of $X$, endowed with the Hausdorff metric $d_{H}$. We prove that each connected component $\mathcal{H}$ of the $\operatorname{space} \operatorname{Conv}_{H}(X)$ is homeomorphic to one of the spaces: $\{0\}, \mathbb{R}, \mathbb{R} \times \mathbb{R}_{+}, Q \times \mathbb{R}_{+}, l_{2}$, or the Hilbert space $l_{2}(\kappa)$ of cardinality $\kappa \geq \mathfrak{c}$. More precisely, a component $\mathcal{H}$ of $\operatorname{Conv}_{\mathrm{H}}(X)$ is homeomorphic to:

(1) $\{0\}$ iff $\mathcal{H}$ contains the whole space $X$;

(2) $\mathbb{R}$ iff $\mathcal{H}$ contains a half-space;

(3) $\mathbb{R} \times \overline{\mathbb{R}}_{+}$iff $\mathcal{H}$ contains a linear subspace of $X$ of codimension 1 ;

(4) $Q \times \overline{\mathbb{R}}_{+}$iff $\mathcal{H}$ contains a linear subspace of $X$ of finite codimension $\geq 2$;

(5) $l_{2}$ iff $\mathcal{H}$ contains a polyhedral convex subset of $X$ but contains no linear subspace and no half-space in $X$;

(6) $l_{2}(\kappa)$ for some cardinal $\kappa \geq \mathfrak{c}$ iff $\mathcal{H}$ contains no polyhedral convex subset of $X$.
\end{abstract}

\section{INTRODUCTION}

In this paper we recognize the topological structure of the space $\operatorname{Conv}_{\mathrm{H}}(X)$ of non-empty closed convex subsets of a Banach space $X$. The space $\operatorname{Conv}_{\mathrm{H}}(X)$ is endowed with the Hausdorff metric

$$
\mathrm{d}_{\mathrm{H}}(A, B)=\max \left\{\sup _{a \in A} \operatorname{dist}(a, B), \sup _{b \in B} \operatorname{dist}(b, A)\right\} \in[0, \infty]
$$

where $\operatorname{dist}(a, B)=\inf _{b \in B}\|a-b\|$ is the distance from a point $a$ to a subset $B$ in $X$. In fact, the topology of $\operatorname{Conv}_{\mathrm{H}}(X)$ can be defined directly without appealing to the Hausdorff metric: a subset $\mathcal{U} \subset \operatorname{Conv}_{\mathrm{H}}(X)$ is open if and only if for every $A \in \mathcal{U}$ there is an open neighborhood $U$ of the origin in $X$ such that $B(A, U) \subset \mathcal{U}$ where $B(A, U)=\left\{A^{\prime} \in \operatorname{Conv}_{\mathrm{H}}(X): A^{\prime} \subset A+U\right.$ and $\left.A \subset A^{\prime}+U\right\}$. Here as usual, $A+B=\{a+b: a \in A, b \in B\}$ stands for the pointwise sum of sets $A, B \subset X$. In such a way, for every linear topological space $X$ we can define the topology on the space $\operatorname{Conv}_{\mathrm{H}}(X)$ of non-empty closed convex subsets of $X$. This topology will be called the uniform topology on $\operatorname{Conv}_{\mathrm{H}}(X)$ because it is generated by the uniformity whose base consists of the sets

$$
2^{U}=\left\{\left(A, A^{\prime}\right) \in \operatorname{Conv}_{\mathrm{H}}(X)^{2}: A \subset A^{\prime}+U, A^{\prime} \subset A+U\right\}
$$

where $U$ runs over open symmetric neighborhoods of the origin in $X$.

We shall observe in Remark 4.8 that for a Banach space $X$ the space $\operatorname{Conv}_{\mathrm{H}}(X)$ is locally connected: two sets $A, B \in \operatorname{Conv}_{\mathrm{H}}(X)$ lie in the same connected component of $\operatorname{Conv}_{\mathrm{H}}(X)$ if and only if $\mathrm{d}_{\mathrm{H}}(A, B)<+\infty$. So, in order to understand the topological structure of the hyperspace $\operatorname{Conv}_{\mathrm{H}}(X)$ it suffices to recognize the topology of its connected components. This problem is quite easy if $X$ is a 1-dimensional real space. In this case $X$ is isometric to $\mathbb{R}$ and a connected component $\mathcal{H}$ of $\operatorname{Conv}_{\mathrm{H}}(X)$ is isometric to:

(1) $\{0\}$ iff $X \in \mathcal{H}$;

(2) $\mathbb{R}$ iff $\mathcal{H}$ contains a closed ray;

(3) $\mathbb{R} \times \overline{\mathbb{R}}_{+}$iff $\mathcal{H}$ contains a bounded set.

Here $\overline{\mathbb{R}}_{+}=[0,+\infty)$ stands for the closed half-line.

For arbitrary Banach spaces we shall add to this list two more spaces:

(4) $Q \times \overline{\mathbb{R}}_{+}$, where $Q=[0,1]^{\omega}$ is the Hilbert cube;

(5) $l_{2}(\kappa)$, the Hilbert space having an orthonormal basis of cardinality $\kappa$.

For $\kappa=\omega$ the separable Hilbert space $l_{2}(\omega)$ is usually denoted by $l_{2}$. By the famous Toruńczyk Theorem [15, 16], each infinite-dimensional Banach space $X$ of density $\kappa$ is homeomorphic to the Hilbert space $l_{2}(\kappa)$. In particular, the Banach space $l_{\infty}$ of bounded real sequences is homeomorphic to $l_{2}(\mathfrak{c})$. In the sequel we shall identify cardinals with the sets of ordinals of smaller cardinality and endow such sets with discrete topology. The cardinality of a set $A$ is denoted by $|A|$.

Let $X$ be a Banach space. As we shall see in Theorem 1, each non-locally compact connected component $\mathcal{H}$ of the space $\operatorname{Conv}_{H}(X)$ is homeomorphic to the Hilbert spaces $l_{2}(\kappa)$ of density $\kappa=\operatorname{dens}(\mathcal{H})$. This reduces the

1991 Mathematics Subject Classification. 57N20; 46A55; 46B26; 46B20; 52B05; 03E65.

Key words and phrases. Banach space, space of closed convex sets, Hilbert space, density, Kunen-Shelah property, polyhedral convex set. 
problem of recognizing the topology of $\operatorname{Conv}_{\mathrm{H}}(X)$ to calculating the densities of its components. In fact, separable components $\mathcal{H}$ of $\operatorname{Conv}_{\mathbf{H}}(X)$ have been characterized in [3] as components containing a polyhedral convex set.

We recall that a convex subset $C$ of a Banach space $X$ is polyhedral if $C$ can be written as the intersection $C=\bigcap \mathcal{F}$ of a finite family $\mathcal{F}$ of closed half-spaces. A half-space in $X$ is a convex set of the form $f^{-1}((-\infty, a])$ for some real number $a$ and some non-zero linear continuous functional $f: X \rightarrow \mathbb{R}$. The whole space $X$ is polyhedral being the intersection $X=\bigcap \mathcal{F}$ of the empty family $\mathcal{F}=\emptyset$ of closed half-spaces.

The principal result of this paper is the following classification theorem.

Theorem 1. Let $X$ be a Banach space. Each connected component $\mathcal{H}$ of the space $\operatorname{Conv}_{\mathrm{H}}(X)$ is homeomorphic to one of the spaces: $\{0\}, \mathbb{R}, \mathbb{R} \times \overline{\mathbb{R}}_{+}, Q \times \overline{\mathbb{R}}_{+}, l_{2}$, or the Hilbert space $l_{2}(\kappa)$ of density $\kappa \geq \mathfrak{c}$. More precisely, $\mathcal{H}$ is homeomorphic to:

(1) $\{0\}$ iff $\mathcal{H}$ contains the whole space $X$;

(2) $\mathbb{R}$ iff $\mathcal{H}$ contains a half-space;

(3) $\mathbb{R} \times \overline{\mathbb{R}}_{+}$iff $\mathcal{H}$ contains a linear subspace of $X$ of codimension 1 ;

(4) $Q \times \overline{\mathbb{R}}_{+}$iff $\mathcal{H}$ contains a linear subspace of $X$ of finite codimension $\geq 2$;

(5) $l_{2}$ iff $\mathcal{H}$ contains a polyhedral convex subset of $X$ but contains no linear subspace and no half-space in $X$;

(6) $l_{2}(\kappa)$ for some cardinal $\kappa \geq \mathfrak{c}$ iff $\mathcal{H}$ contains no polyhedral convex subset of $X$.

Theorem 1 will be proved in Section 6 after some preliminary work done in Sections 25 .

In Corollary 2 below we shall derive from Theorem 1 a complete topological classification of the spaces $\operatorname{Conv}_{\mathrm{H}}(X)$ for Banach spaces $X$ with Kunen-Shelah property and $\left|X^{*}\right| \leq \mathfrak{c}$.

A Banach space $X$ is defined to have the Kunen-Shelah property if each closed convex subset $C \subset X$ can be written as intersection $C=\bigcap \mathcal{F}$ of an at most countable family $\mathcal{F}$ of closed half-spaces (in fact, this is one of seven equivalent Kunen-Shelah properties considered in [6] and [7, 8.19]). For a Banach space $X$ with the Kunen-Shelah property we get

$$
\left|X^{*}\right| \leq\left|\operatorname{Conv}_{H}(X)\right| \leq\left|X^{*}\right|^{\omega} .
$$

The upper bound $\operatorname{Conv}_{\mathrm{H}}(X) \leq\left|X^{*}\right|^{\omega}$ follows from the definition of the Kunen-Shelah property while the lower bound $\left|X^{*}\right| \leq\left|\operatorname{Conv}_{\mathrm{H}}(X)\right|$ follows from the observation that a functional $f \in X^{*}$ is uniquely determined by its polar half-space $H_{f}=f^{-1}((-\infty, 1])$.

It is clear that each separable Banach space has the Kunen-Shelah property. However there are also non-separable Banach spaces with that property. The first example of such Banach space was constructed by S. Shelah under $\nabla_{\aleph_{1}}$ [13. The second example is due to K. Kunen who used the Continuum Hypothesis to construct a non-metrizable scattered compact space $K$ such that the Banach space $X=C(K)$ of continuous functions on $K$ is hereditarily Lindelöf in the weak topology and thus has the Kunen-Shelah Property, see [10, p.1123]. The Kunen's space $X=C(K)$ has an additional property that its dual space $X^{*}=C(X)^{*}$ has cardinality $\left|X^{*}\right|=\mathfrak{c}$ (this follows from the fact that each Borel measure on the scattered compact space $K$ has countable support). Let us remark that for every separable Banach space $X$ the dual space $X^{*}$ also has cardinality of continuum $\left|X^{*}\right|=\mathfrak{c}$. It should be mentioned that non-separable Banach spaces with the Kunen-Shelah property can be constructed only under certain additional set-theoretic assumptions. By [14, there are models of ZFC in which each Banach space with the Kunen-Shelah property is separable.

Corollary 1. For a separable Banach space (more generally, a Banach space with the Kunen-Shelah property and $\left.\left|X^{*}\right|=\mathfrak{c}\right)$, each connected component $\mathcal{H}$ of the space $\operatorname{Conv}_{\mathrm{H}}(X)$ is homeomorphic to $\{0\}, \mathbb{R}, \mathbb{R} \times \overline{\mathbb{R}}_{+}, Q \times \overline{\mathbb{R}}_{+}, l_{2}$ or $l_{\infty}$. More precisely, $\mathcal{H}$ is homeomorphic to:

(1) $\{0\}$ iff $\mathcal{H}$ contains the whole space $X$;

(2) $\mathbb{R}$ iff $\mathcal{H}$ contains a half-space;

(3) $\mathbb{R} \times \overline{\mathbb{R}}_{+}$iff $\mathcal{H}$ contains a linear subspace of $X$ of codimension 1 ;

(4) $Q \times \overline{\mathbb{R}}_{+}$iff $\mathcal{H}$ contains a linear subspace of $X$ of codimension $\geq 2$;

(5) $l_{2}$ iff $\mathcal{H}$ contains a polyhedral convex set but contains no linear subspace and no half-space;

(6) $l_{\infty}$ iff $\mathcal{H}$ contains no polyhedral convex set.

Since the space $\operatorname{Conv}_{\mathrm{H}}(X)$ is homeomorphic to the topological sum of its connected components, we can use Corollary 1 to classify topologically the spaces $\operatorname{Conv}_{\mathrm{H}}(X)$ for separable Banach spaces $X$ (and more generally Banach spaces with the Kunen-Shelah property and $\left|X^{*}\right| \leq \mathfrak{c}$ ). In the following corollary the cardinal $\mathfrak{c}$ is considered as a discrete topological space.

Corollary 2. For a separable Banach space $X$ (more generally, a Banach space $X$ with the Kunen-Shelah property and $\left.\left|X^{*}\right| \leq \mathfrak{c}\right)$ the space $\operatorname{Conv}_{\mathrm{H}}(X)$ is homeomorphic to the topological sum:

(1) $\{0\} \oplus \mathbb{R} \oplus \mathbb{R} \oplus\left(\mathbb{R} \times \overline{\mathbb{R}}_{+}\right)$iff $\operatorname{dim}(X)=1$;

(2) $\{0\} \oplus Q \times \overline{\mathbb{R}}_{+} \oplus \mathfrak{c} \times\left(\mathbb{R} \oplus \mathbb{R} \times \overline{\mathbb{R}}_{+} \oplus l_{2} \oplus l_{\infty}\right)$ iff $\operatorname{dim}(X)=2$; 
(3) $\{0\} \oplus \mathfrak{c} \times\left(\mathbb{R} \oplus \mathbb{R} \times \overline{\mathbb{R}}_{+} \oplus Q \times \overline{\mathbb{R}}_{+} \oplus l_{2} \oplus l_{\infty}\right)$ iff $\operatorname{dim}(X) \geq 3$.

Moreover, under $2^{\omega_{1}}>\mathfrak{c}$, for a Banach space $X$, the space $\operatorname{Conv}_{\mathrm{H}}(X)$ has cardinality $\left|\operatorname{Conv}_{\mathrm{H}}(X)\right| \leq \mathfrak{c}$ if and only if $\left|X^{*}\right| \leq \mathfrak{c}$ and the Banach space $X$ has the Kunen-Shelah property.

Proof. The statements (1)-(3) easily follow from the classification of the components of $\operatorname{Conv}_{H}(X)$ given in Corollary 1 and a routine calculation of the number of components of a given topological type.

Now assume that $2^{\omega_{1}}>\mathfrak{c}$. If $X$ is a Banach space with the Kunen-Shelah property and $\left|X^{*}\right| \leq \mathfrak{c}$, then the definition of the Kunen-Shelah property yields the upper bound

$$
\left|\operatorname{Conv}_{\mathrm{H}}(X)\right| \leq\left|X^{*}\right|^{\omega} \leq \mathfrak{c}^{\omega}=\mathfrak{c} .
$$

If $\left|\operatorname{Conv}_{\mathrm{H}}(X)\right| \leq \mathfrak{c}$, then $\left|X^{*}\right| \leq \mathfrak{c}$ as $\left|X^{*}\right| \leq\left|\operatorname{Conv}_{\mathrm{H}}(X)\right|$ (because each functional $f \in X^{*}$ can be uniquely identified with its polar half-space $\left.f^{-1}((-\infty, 1]) \in \operatorname{Conv}_{H}(X)\right)$. Assuming that $X$ fails to have the Kunen-Shelah property and applying Theorem 8.19 of [7] (see also [6]), we can find a sequence $\left\{x_{\alpha}\right\}_{\alpha<\omega_{1}} \subset X$ such that for every $\alpha<\omega_{1}$ the point $x_{\alpha}$ does not lie in the closed convex hull $C_{\omega_{1} \backslash\{\alpha\}}$ of the set $\left\{x_{\alpha}\right\}_{\alpha \in \omega_{1} \backslash\{\alpha\}}$. Now for every subset $A \subset \omega_{1}$ consider the closed convex hull $C_{A}=\overline{\operatorname{conv}}\left\{x_{\alpha}\right\}_{\alpha \in A}$. We claim that $C_{A} \neq C_{B}$ for any distinct subsets $A, B \subset \omega_{1}$. Indeed, if $A \neq B$ then the symmetric difference $(A \backslash B) \cup(B \backslash A)$ contains some ordinal $\alpha$. Without loss of generality, we can assume that $\alpha \in A \backslash B$. Then $x_{\alpha} \in C_{A} \backslash C_{B}$ as $C_{B} \subset C_{\omega_{1} \backslash\{\alpha\}} \ni x_{\alpha}$. This implies that $\left\{C_{A}: A \subset \omega_{1}\right\}$ is a subset of cardinality $2^{\omega_{1}}>\mathfrak{c}$ in $\operatorname{Conv}_{\mathrm{H}}(X)$ and hence $\left|\operatorname{Conv}_{\mathrm{H}}(X)\right| \geq 2^{\omega_{1}}>\mathfrak{c}$, which is a desired contradiction.

Among the connected components of $\operatorname{Conv}_{\mathrm{H}}(X)$ there is a special one, namely, the component $\mathcal{H}_{0}$ containing the singleton $\{0\}$. This component coincides with the space $\operatorname{BConv}_{H}(X)$ of all non-empty bounded closed convex subsets of a Banach space $X$. The spaces $\operatorname{BConv}_{H}(X)$ have been intensively studied both by topologists [9], 12 , and analysts [5. In particular, S.Nadler, J. Quinn and N.M. Stavrakas 9] proved that for a finite $n \geq 2$ the space $\operatorname{BConv}_{H}\left(\mathbb{R}^{n}\right)$ is homeomorphic to $Q \times \overline{\mathbb{R}}_{+}$while K. Sakai proved in 12 that for an infinite-dimensional Banach space $X$ the space $\mathcal{H}_{0}=\operatorname{BConv}_{H}(X)$ is homeomorphic to a non-separable Hilbert space. Moreover, if $X$ is separable or reflexive, then $\operatorname{dens}\left(\mathcal{H}_{0}\right)=2^{\operatorname{dens}(X)}$. In the latter case the density dens ${ }^{*}\left(X^{*}\right)$ of the dual space $X^{*}$ in the weak* topology is equal to the density $\operatorname{dens}(X)$ of $X$. Banach spaces $X$ with $\operatorname{dens}^{*}\left(X^{*}\right)=\operatorname{dens}\left(X^{*}\right)$ are called DENS Banach spaces, see [7, 5.39]. By Proposition 5.40 of [7], the class of DENS Banach spaces includes all weakly Linedlöf determined spaces, and hence all weakly countably generated and all reflexive Banach spaces.

Applying Theorem 1 to describing the topology of the component $\mathcal{H}_{0}=\operatorname{BConv}_{H}(X)$, we obtain the following classification.

Corollary 3. The space $\mathcal{H}_{0}=\mathrm{BConv}_{H}(X)$ of non-empty bounded closed convex subsets of a Banach space $X$ is homeomorphic to one of the spaces: $\{0\}, \mathbb{R} \times \overline{\mathbb{R}}_{+}, Q \times \overline{\mathbb{R}}_{+}$or the Hilbert space $l_{2}(\kappa)$ of density $\kappa \geq \mathfrak{c}$. More precisely, $\operatorname{BConv}(X)$ is homeomorphic to:

(1) $\{0\}$ iff $\operatorname{dim} X=0$;

(2) $\mathbb{R} \times \mathbb{R}^{+}$iff $\operatorname{dim} X=1$;

(3) $Q \times \overline{\mathbb{R}}_{+}$iff $2 \leq \operatorname{dim}(X)<\infty$;

(4) $l_{2}(\kappa)$ for some cardinal $\kappa \in\left[2^{\operatorname{dens}^{*}\left(X^{*}\right)}, 2^{\operatorname{dens}(X)}\right]$ iff $\operatorname{dim}(X)=\infty$;

(5) $l_{2}\left(2^{\operatorname{dens}(X)}\right)$ if $X$ is an infinite-dimensional DENS Banach space.

Proof. This corollary will follow from Theorem 1 as soon as we check that

$$
2^{\operatorname{dens}^{*}\left(X^{*}\right)} \leq \operatorname{dens}\left(\mathcal{H}_{0}\right) \leq\left|\mathcal{H}_{0}\right| \leq\left|\operatorname{Conv}_{H}(X)\right| \leq 2^{\operatorname{dens}(X)}
$$

for each infinite-dimensional Banach space $X$.

In fact, the inequality $\left|\operatorname{Conv}_{\mathrm{H}}(X)\right| \leq 2^{\operatorname{dens}(X)}$ has general-topological nature and follows from the known fact that the number of closed subsets (equal to the number of open subsets) of a topological space $X$ does not exceed $2^{w(X)}$.

To prove that $2^{\text {dens }^{*}\left(X^{*}\right)} \leq \operatorname{dens}\left(\mathcal{H}_{0}\right)$ we shall use a result of Plichko [11] (see also Theorem 4.12 [7]) saying that for each infinite-dimensional Banach space $X$ there is a bounded sequence $\left\{\left(x_{\alpha}, f_{\alpha}\right)\right\}_{\alpha<\kappa} \subset X \times X^{*}$ of length $\kappa=$ dens $^{*}\left(X^{*}\right)$, which is biorthogonal in the sense that $f_{\alpha}\left(x_{\alpha}\right)=1$ and $f_{\alpha}\left(x_{\beta}\right)=0$ for any distinct ordinals $\alpha, \beta<\kappa$. Let $L=\sup \left\{\left\|x_{\alpha}\right\|,\left\|f_{\alpha}\right\|: \alpha<\kappa\right\}$.

For every subset $A \subset \kappa$ consider the closed convex hull $C_{A}=\overline{\operatorname{conv}}\left(\left\{x_{\alpha}\right\}_{\alpha \in A}\right)$ of the set $\left\{x_{\alpha}\right\}_{\alpha \in A}$. We claim that for any distinct subsets $A, B \subset \kappa$ we get $\mathrm{d}_{\mathrm{H}}\left(C_{A}, C_{B}\right) \geq \frac{1}{L}$. Indeed, since $A \neq B$ the symmetric difference $(A \backslash B) \cup(B \backslash A)$ contains some ordinal $\alpha$. Without loss of generality, we can assume that $\alpha \in A \backslash B$. Then $C_{B} \subset f^{-1}(0)$ and hence for each $c \in C_{B}$ we get $\left\|x_{\alpha}-c\right\| \geq \frac{\left|f_{\alpha}\left(x_{\alpha}\right)-f_{\alpha}(c)\right|}{\left\|f_{\alpha}\right\|} \geq \frac{|1-0|}{L}$, which implies $\operatorname{dist}\left(x_{\alpha}, C_{B}\right) \geq \frac{1}{L}$ and hence $\mathrm{d}_{\mathrm{H}}\left(C_{A}, C_{B}\right) \geq \frac{1}{L}$ as $x_{\alpha} \in C_{A}$.

Now we see that $\mathcal{C}=\left\{C_{A}: A \subset \kappa\right\}$ is a closed discrete subspace in $\mathcal{H}_{0}$ and hence $\operatorname{dens}\left(\mathcal{H}_{0}\right) \geq|\mathcal{C}|=2^{\kappa}=$ $2^{\mathrm{dens}^{*}(X *)}$. 
Corollaries 1 and 2 motivate the following problem.

Problem 1.1. Is $\left|X^{*}\right| \leq \mathfrak{c}$ for each Banach space $X$ with the Kunen-Shelah property?

Another problem concerns possible densities of the components of the space $\mathrm{Conv}_{\mathrm{H}}(X)$.

Problem 1.2. Let $X$ be an infinite-dimensional Banach space. Is it true that each component $\mathcal{H}$ (in particular,



Observe that under GCH (the Generalized Continuum Hypothesis) the answer to Problem 1.2 is trivially "yes" as under GCH all cardinals are of the form $2^{<\kappa}$ for some $\kappa$.

\section{HYPERMETRIC SPACES}

Because the Hausdorff distance $\mathrm{d}_{\mathrm{H}}$ on $\operatorname{Conv}_{\mathrm{H}}(X)$ can take the infinite value we should work with generalized metrics called hypermetrics. The precise definition is as follows:

A hypermetric on a set $X$ is a function $d: X \times X \rightarrow[0, \infty]$ satisfying the three axioms of a usual metric:

- $d(x, y)=0$ iff $x=y$,

- $d(x, y)=d(y, x)$

- $d(x, z) \leq d(x, y)+d(y, z)$.

Here we extend the addition operation from $(-\infty, \infty)$ to $[-\infty, \infty]$ letting

$$
\infty+\infty=\infty,-\infty+(-\infty)=-\infty, \infty+(-\infty)=-\infty+\infty=0
$$

and

for every $x \in(-\infty, \infty)$.

$$
x+\infty=\infty+x=\infty, x+(-\infty)=-\infty+x=-\infty
$$

A hypermetric space is a pair $(X, d)$ consisting of a set $X$ and a hypermetric $d$ on $X$. It is clear that each metric is a hypermetric and hence each metric space is a hypermetric space.

In some respect, the notion of a hypermetric is more convenient than the usual notion of a metric. In particular, for any family $\left(X_{i}, d_{i}\right), i \in \mathcal{I}$, of hypermetric spaces it is trivial to define a nice hypermetric $d$ on the topological $\operatorname{sum} X=\oplus_{i \in \mathcal{I}} X_{i}$. Just let

$$
d(x, y)= \begin{cases}d_{i}(x, y), & \text { if } x, y \in X_{i} \\ \infty, & \text { otherwise }\end{cases}
$$

The obtained hypermetric space $(X, d)$ will be called the direct sum of the family of hypermetric spaces $\left(X, d_{i}\right)$, $i \in \mathcal{I}$.

In fact, each hypermetric space $(X, d)$ decomposes into the direct sum of metric subspaces of $X$ called metric components of $X$. More precisely, a metric component of $X$ is an equivalence class of $X$ by the equivalence relation $\sim$ defined as $x \sim y$ iff $d(x, y)<\infty$. So, the metric component of a point $x \in X$ coincides with the set $\mathbb{B}_{<\infty}(x)=\left\{x^{\prime} \in X: d\left(x, x^{\prime}\right)<\infty\right\}$. The restriction of the hypermetric $d$ to each metric component is a metric. Therefore $X$ is the direct sum of its metric components and hence understanding the (topological) structure of a hypermetric space reduces to studying the metric (or topological) structure of its metric components.

A typical example of a hypermetric is the Hausdorff hypermetric $d_{H}$ on the space $\operatorname{Cld}(X)$ of non-empty closed subsets of a (linear) metric space $X$ (and the restriction of $\mathrm{d}_{\mathrm{H}}$ to the subspace $\operatorname{Conv}(X) \subset \operatorname{Cld}(X)$ of non-empty closed convex subsets of $X)$. So both $\operatorname{Cld}_{\mathrm{H}}(X)=\left(\operatorname{Cld}(X), \mathrm{d}_{\mathrm{H}}\right)$ and $\operatorname{Conv}_{\mathrm{H}}(X)=\left(\operatorname{Conv}(X), \mathrm{d}_{\mathrm{H}}\right)$ are hypermetric spaces.

A much simple (but still important) example of a hypermetric space is the extended real line $\overline{\mathbb{R}}=[-\infty, \infty]$ with the hypermetric

$$
d(x, y)= \begin{cases}|x-y|, & \text { if } x, y \in(-\infty, \infty), \\ 0, & \text { if } x=y \in\{-\infty, \infty\} \\ \infty, & \text { otherwise }\end{cases}
$$

which will be denoted by $|x-y|$. The hypermetric space $\overline{\mathbb{R}}$ has three metric components: $\{-\infty\}, \mathbb{R},\{\infty\}$.

This example allows us to construct another important example of a hypermetric space. Namely, for a set $\Gamma$ consider the space $\overline{\mathbb{R}}^{\Gamma}$ of functions from $\Gamma$ to $\overline{\mathbb{R}}$ endowed with the hypermetric

$$
d(f, g)=\|f-g\|_{\infty}=\sup _{\gamma \in \Gamma}|f(\gamma)-g(\gamma)| .
$$

The obtained hypermetric space $\left(\overline{\mathbb{R}}^{\Gamma},\|\cdot-\cdot\|_{\infty}\right)$ will be denoted by $\bar{l}_{\infty}(\Gamma)$. Observe that the topology of $\bar{l}_{\infty}(\Gamma)$ is different from the Tychonoff product topology of $\bar{R}$. Another reason for using the notation $\bar{l}_{\infty}(\Gamma)$ is that the 
metric component of $\bar{l}_{\infty}(\Gamma)$ containing the zero function coincides with the classical Banach space $l_{\infty}(\Gamma)$ of bounded functions on $\Gamma$. More generally, for each $f_{0} \in \bar{l}_{\infty}(\Gamma)$ its metric component

$$
\mathbb{B}_{<\infty}\left(f_{0}\right)=\left\{f \in \bar{l}_{\infty}(\Gamma):\left\|f-f_{0}\right\|_{\infty}<\infty\right\}
$$

is isometric to the Banach space $l_{\infty}\left(\Gamma_{0}\right)$ where $\Gamma_{0}=\left\{\gamma \in \Gamma:\left|f_{0}(\gamma)\right|<\infty\right\}$.

It turns out that for every normed space $X$ the space $\operatorname{Conv}_{H}(X)$ nicely embeds into the hypermetric space $\bar{l}_{\infty}\left(\mathbb{S}^{*}\right)$ where

$$
\mathbb{S}^{*}=\left\{x^{*} \in X^{*}:\left\|x^{*}\right\|=1\right\}
$$

stands for the unit sphere of the dual Banach space $X^{*}$.

Namely, consider the function

$$
\delta: \operatorname{Conv}_{\mathrm{H}}(X) \mapsto \bar{l}_{\infty}\left(\mathbb{S}^{*}\right), \delta: C \mapsto \delta_{C}
$$

where $\delta_{C}\left(x^{*}\right)=\sup x^{*}(C)$ for $x^{*} \in \mathbb{S}^{*}$. The function $\delta$ will be called the canonical representation of $\operatorname{Conv}_{\mathrm{H}}(X)$.

Proposition 2.1. For every normed space $X$ the canonical representation $\delta: \operatorname{Conv}_{\mathrm{H}}(X) \rightarrow \bar{l}_{\infty}\left(\mathbb{S}^{*}\right)$ is an isometric embedding.

Proof. Let $A, B \in \operatorname{Conv}_{\mathrm{H}}(X)$ be two convex sets. We should prove that $\mathrm{d}_{\mathrm{H}}(A, B)=\left\|\delta_{A}-\delta_{B}\right\|$, where

$$
\left\|\delta_{A}-\delta_{B}\right\|=\sup _{x^{*} \in \mathbb{S}^{*}}\left|\delta_{A}\left(x^{*}\right)-\delta_{B}\left(x^{*}\right)\right|=\sup _{x^{*} \in \mathbb{S}^{*}}\left|\sup x^{*}(A)-\sup x^{*}(B)\right| .
$$

The inequality $\left\|\delta_{A}-\delta_{B}\right\| \leq \mathrm{d}_{\mathrm{H}}(A, B)$ will follow as soon as we check that $\left|\sup x^{*}(A)-\sup x^{*}(B)\right| \leq \mathrm{d}_{\mathrm{H}}(A, B)$ for each functional $x^{*} \in \mathbb{S}^{*}$. This is trivial if $\mathrm{d}_{\mathrm{H}}(A, B)=\infty$. So we assume that $\mathrm{d}_{\mathrm{H}}(A, B)<\infty$. To obtain a contradiction, assume that $\left|\sup x^{*}(A)-\sup x^{*}(B)\right|>\mathrm{d}_{\mathrm{H}}(A, B)$. Then either $\sup x^{*}(A)-\sup x^{*}(B)>\mathrm{d}_{\mathrm{H}}(A, B)$ or $\sup x^{*}(B)-\sup x^{*}(A)>\mathrm{d}_{\mathrm{H}}(A, B)$. In the first case $\sup x^{*}(B) \neq \infty$, so we can find a point $a \in A$ with $x^{*}(a)-\sup x^{*}(B)>\mathrm{d}_{\mathrm{H}}(A, B)$. It follows from the definition of the Hausdorff metric $\mathrm{d}_{\mathrm{H}}(A, B) \geq \operatorname{dist}(a, B)$ that $\|a-b\|<x^{*}(a)-\sup x^{*}(B)$ for some point $b \in B$. Then $x^{*}(a)-x^{*}(b) \leq\left\|x^{*}\right\| \cdot\|a-b\|<x^{*}(a)-\sup x^{*}(B)$ and hence $x^{*}(b)>\sup x^{*}(B)$, which is a contradiction.

By analogy, we can derive a contradiction from the assumption $\sup x^{*}(B)-\sup x^{*}(A)>\mathrm{d}_{\mathrm{H}}(A, B)$ and thus prove the inequality $\left\|\delta_{A}-\delta_{B}\right\| \leq \mathrm{d}_{\mathrm{H}}(A, B)$.

To prove the reverse inequality $\left\|\delta_{A}-\delta_{B}\right\| \geq \mathrm{d}_{\mathrm{H}}(A, B)$ let us consider two cases:

(i) $\mathrm{d}_{\mathrm{H}}(A, B)=\infty$. To prove that $\infty=\left\|\delta_{A}-\delta_{B}\right\|$, it suffices given any number $R<\infty$ to find a linear functional $x^{*} \in \mathbb{S}^{*}$ such that $\left|\sup x^{*}(A)-\sup x^{*}(B)\right| \geq R$.

The equality $\mathrm{d}_{\mathrm{H}}(A, B)=\infty$ implies that either $\sup _{a \in A} \operatorname{dist}(a, B)=\infty$ or $\sup _{b \in B} \operatorname{dist}(b, A)=\infty$. In the first case we can find a point $a \in A$ with $\operatorname{dist}(a, B) \geq R$ and using the Hahn-Banach Theorem construct a linear functional $x^{*} \in \mathbb{S}^{*}$ that separates the convex set $B$ from the closed $R$-ball $\bar{B}(a, R)=\{x \in X:\|x-a\| \leq R\}$ in the sense that $\sup x^{*}(B) \leq \inf x^{*}(\bar{B}(a, R))$. For this functional $x^{*}$ we get $\sup x^{*}(A) \geq x^{*}(a) \geq R+\inf x^{*}(\bar{B}(a, R)) \geq R+\sup x^{*}(B)$ and thus $\sup x^{*}(A)-\sup x^{*}(B) \geq R$.

In the second case, we can repeat the preceding argument to find a linear functional $x^{*} \in \mathbb{S}^{*}$ with

$$
\left|\sup x^{*}(A)-\sup x^{*}(B)\right| \geq \sup x^{*}(B)-\sup x^{*}(X) \geq R \text {. }
$$

(ii) $\mathrm{d}_{\mathrm{H}}(A, B)<\infty$. To prove that $\mathrm{d}_{\mathrm{H}}(A, B) \geq\left\|\delta_{A}-\delta_{B}\right\|$ it suffices given any number $\varepsilon>0$ to find a linear functional $x^{*} \in \mathbb{S}^{*}$ such that $\left|\sup x^{*}(A)-\sup x^{*}(B)\right| \geq \mathrm{d}_{\mathrm{H}}(A, B)-\varepsilon$. It follows from the definition of $\mathrm{d}_{\mathrm{H}}(A, B)$ that either there is a point $a \in A$ with $\operatorname{dist}(a, B)>\mathrm{d}_{\mathrm{H}}(A, B)-\varepsilon$ or else there is a point $b \in B$ with $\operatorname{dist}(b, A)>\mathrm{d}_{\mathrm{H}}(A, B)-\varepsilon$. In the first case we an use the Hahn-Banach Theorem to find a linear functional $x^{*} \in \mathbb{S}^{*}$ which separates the convex set $B$ from the closed $R$-ball $\bar{B}(a, R)$ where $R=\mathrm{d}_{\mathrm{H}}(A, B)-\varepsilon$ in the sense that $\sup x^{*}(B) \leq \inf x^{*}(\bar{B}(a, R)$. Then

$$
\sup x^{*}(B) \leq \inf x^{*}(\bar{B}(a, R))=x^{*}(a)-R \leq \sup x^{*}(A)-R
$$

and hence

$$
\left|\sup x^{*}(A)-\sup x^{*}(B)\right| \geq \sup x^{*}(A)-\sup x^{*}(B) \geq R=\mathrm{d}_{\mathrm{H}}(A, B)-\varepsilon .
$$

The second case can be considered by analogy.

\section{Assigning cones to Components of $\operatorname{Conv}_{\mathrm{H}}(X)$}

In this section to each convex set $C$ of a normed space $X$ we assign two cones: the characteristic cone $V_{C} \subset X$ and the dual characteristic cone $V_{C}^{*} \subset X^{*}$.

We recall that a subset $V$ of a linear space $L$ is called a convex cone if $a x+b y \in V$ for any points $x, y \in W$ and non-negative real numbers $a, b \in[0,+\infty)$.

For a convex subset $C$ of a normed space $X$ its characteristic cone of $C$ is the convex cone

$$
V_{C}=\left\{v \in X: \forall c \in C, c+\overline{\mathbb{R}}_{+} v \subset c\right\}
$$


lying in the normed space $X$, and its dual characteristic cone $V_{C}^{*}$ is a closed convex cone

$$
V_{C}^{*}=\left\{x^{*} \in X^{*}: \sup x^{*}(C)<\infty\right\}
$$

which lies in the dual Banach space $X^{*}$.

It turns out that the characteristic cone $V_{C}$ of a convex set $C$ is uniquely determined by its dual characteristic cone $V_{C}^{*}$.

Lemma 3.1. For any non-empty closed convex set $C$ in a normed space $X$ we get

$$
V_{C}=\bigcap_{f \in V_{C}^{*}} f^{-1}((-\infty, 0]) .
$$

Proof. Fix any vector $v \in V_{C}$ and a functional $f \in V_{C}^{*}$. Observe that for each number $t \in \overline{\mathbb{R}}_{+}$, we get $c+t v \in$ $C$ and hence $f(c)+t f(v) \leq \sup f(C)<\infty$, which implies that $f(v) \leq 0$. This proves the inclusion $V_{C} \subset$ $\bigcap_{f \in V_{C}^{*}} f^{-1}((-\infty, 0])$.

To prove the reverse inclusion, fix any vector $v \in X \backslash V_{C}$. Then for some point $c \in C$ and some positive real number $t$ we get $c+t v \notin C$. Using the Hahn-Banach Theorem, find a functional $f \in X^{*}$ that separates the convex set $C$ and the point $x=c+t v$ in the sense that $\sup f(C)<f(c+t v)$. Then $f(c) \leq \sup f(C)<f(c)+t f(v)$ implies that $f(v)>0$ and $v \notin f^{-1}((-\infty, 0])$.

Let $X$ be a normed space. It is easy to see that for each component $\mathcal{H}$ of the space $\operatorname{Conv}_{\mathrm{H}}(X)$ and any two convex sets $A, B \in \mathcal{H}$ we get $C_{A}^{*}=C_{B}^{*}$. In this case Lemma 3.1 implies that $C_{A}=C_{B}$ as well. This allows us to define the characteristic cone $V_{\mathcal{H}}$ and the dual characteristic cone $V_{\mathcal{H}}^{*}$ of the component $\mathcal{H}$ letting $V_{\mathcal{H}}=V_{C}$ and $V_{\mathcal{H}}^{*}=V_{C}^{*}$ for any convex set $C \in \mathcal{H}$. Lemma 3.1 guarantees that

$$
V_{\mathcal{H}}=\bigcap_{f \in V_{\mathcal{H}}^{*}} f^{-1}((-\infty, 0]),
$$

so the characteristic cone $V_{\mathcal{H}}$ of $\mathcal{H}$ is uniquely determined by its dual characteristic cone $V_{\mathcal{H}}^{*}$.

\section{The algebraical structure of $\operatorname{Conv}_{H}(X)$}

In this section given a normed space $X$ we study the algebraic properties of the canonical representation $\delta$ : $\operatorname{Conv}_{\mathrm{H}}(X) \rightarrow \bar{l}_{\infty}\left(\mathbb{S}^{*}\right)$.

Note that the space $\operatorname{Conv}_{\mathrm{H}}(X)$ has a rich algebraic structure: it possesses three interrelated algebraic operations: the multiplication by a real number, the addition, and taking the maximum. More precisely, for a real number $t \in \mathbb{R}$, and convex sets $A, B \in \operatorname{Conv}_{\mathrm{H}}(X)$ let

$$
\begin{aligned}
& t \cdot A=\{t a: a \in A\} \\
& A \oplus B=\overline{A+B} ; \\
& \max \{A, B\}=\overline{\operatorname{conv}}(A \cup B), \text { where } \\
& \overline{\operatorname{conv}}(Y) \text { stands for the closed convex hull of a subset } Y \subset X .
\end{aligned}
$$

The hypermetric space $\overline{\mathbb{R}}$ also has the corresponding three operations (multiplication by a real number, addition and taking maximum) which induces the tree operations on $\bar{l}_{\infty}(\Gamma)=\overline{\mathbb{R}}^{\Gamma}$.

Proposition 4.1. The canonical representation $\delta: \operatorname{Conv}_{\mathrm{H}}(X) \rightarrow \bar{l}_{\infty}\left(\mathbb{S}^{*}\right)$ has the following properties:

(1) $\delta(A \oplus B)=\delta(A)+\delta(B)$;

(2) $\delta(\max \{A, B\})=\max \{\delta(A), \delta(B)\}$;

(3) $\delta(r A)=r \delta(A)$;

for every non-negative real number $r$ and convex sets $A, B \in \operatorname{Conv}_{\mathrm{H}}(X)$.

Proof. The three items of the proposition follow from the three obvious equalities

$$
\begin{aligned}
& \sup x^{*}(A \oplus B)=x^{*}(A+B)=x^{*}(A)+x^{*}(B), \\
& \sup x^{*}(\overline{\operatorname{conv}}(A \cup B))=\sup x^{*}(A \cup B)=\max \left\{\sup x^{*}(A), \sup x^{*}(B)\right\}, \\
& \sup x^{*}(r A)=r \sup x^{*}(A) .
\end{aligned}
$$

holding for every functional $x^{*} \in X^{*}$.

Remark 4.2. Easy examples show that the last item of Proposition 4.1 does not hold for negative real numbers $r$. This means that the operator $\delta: \operatorname{Conv}_{\mathbf{H}}(X) \rightarrow \bar{l}_{\infty}\left(\mathbb{S}^{*}\right)$ is positively homogeneous but not homogeneous. 
The operations of addition and multiplication by a real number allow us to define another important operation on $\operatorname{Conv}_{\mathrm{H}}(X)$ preserved by the canonical representation $\delta$, namely the Minkovski operation

$$
\mu: \operatorname{Conv}_{\mathrm{H}}(X) \times \operatorname{Conv}_{\mathrm{H}}(X) \times[0,1] \rightarrow \operatorname{Conv}_{\mathrm{H}}(X), \mu:(A, B, t) \mapsto(1-t) A \oplus t B
$$

of producing a convex combination. Proposition 4.1 implies that the canonical representation $\delta$ is affine in the sense that

$$
\delta((1-t) A \oplus t B)=(1-t) \delta(A)+t \delta(B)
$$

for every $A, B \in \operatorname{Conv}_{\mathrm{H}}(X)$ and $t \in[0,1]$.

Propositions 2.1 and 4.1 will help us to establish the metric properties of the algebraic operations on $\operatorname{Conv}_{\mathrm{H}}(X)$.

Proposition 4.3. Let $A, B, C, A^{\prime}, B^{\prime} \in \operatorname{Conv}_{\mathrm{H}}(X)$ be five convex sets and $r \in \mathbb{R}$ and $t, t^{\prime} \in[0,1]$ be three real numbers. Then

(1) $\mathrm{d}_{\mathrm{H}}\left(A \oplus B, A^{\prime} \oplus B^{\prime}\right) \leq \mathrm{d}_{\mathrm{H}}\left(A, A^{\prime}\right)+\mathrm{d}_{\mathrm{H}}\left(B, B^{\prime}\right)$;

(2) $\mathrm{d}_{\mathrm{H}}(A \oplus B, A \oplus C)=\mathrm{d}_{\mathrm{H}}(B, C)$ provided $V_{A}^{*} \supset V_{B}^{*} \cup V_{C}^{*}$;

(3) $\mathrm{d}_{\mathrm{H}}\left(\max \{A, B\}, \max \left\{A^{\prime}, B^{\prime}\right\}\right) \leq \max \left\{\mathrm{d}_{\mathrm{H}}\left(A, A^{\prime}\right), \mathrm{d}_{\mathrm{H}}\left(B, B^{\prime}\right)\right\}$;

(4) $\mathrm{d}_{\mathrm{H}}(r \cdot A, r \cdot B)=|r| \cdot \mathrm{d}_{\mathrm{H}}(A, B)$;

(5) $\mathrm{d}_{\mathrm{H}}\left((1-t) A \oplus t B,\left(1-t^{\prime}\right) A \oplus t^{\prime} B\right)=\left|t-t^{\prime}\right| \mathrm{d}_{\mathrm{H}}(A, B)$.

Proof. All the items easily follow from Propositions 2.1, 4.1, and metric properties of algebraic operations on the hypermetric space $\bar{l}_{\infty}\left(\mathbb{S}^{*}\right)$.

Observe that the metric components of the hypermetric space $\bar{l}_{\infty}\left(\mathbb{S}^{*}\right)$ are closed with respect to taking maximum and producing a convex combination. Moreover those operations are continuous on metric components of $\bar{l}_{\infty}\left(\mathbb{S}^{*}\right)$. With help of the canonical representation those properties of $\bar{l}_{\infty}\left(\mathbb{S}^{*}\right)$ transform into the corresponding properties of $\operatorname{Conv}_{\mathrm{H}}(X)$. In such a way we obtain

Corollary 4.4. Each metric component $\mathcal{H}$ of $\operatorname{Conv}_{\mathrm{H}}(X)$ is closed under the operations of taking maximum and producing a convex combination. Moreover those operations are continuous on $\mathcal{H}$.

Corollary 4.5. Each metric component $\mathcal{H}$ of $\operatorname{Conv}_{\mathrm{H}}(X)$ is isometric to a convex max-subsemilattice of the Banach lattice $l_{\infty}\left(\mathbb{S}^{*}\right)$.

A subset of a Banach lattice is called a max-subsemilattice is it is closed under the operation of taking maximum.

By a recent result of Banakh and Cauty [1, each non-locally compact closed convex subset of a Banach space is homeomorphic to an infinite-dimensional Hilbert space. This result combined with Corollary 4.5 implies:

Corollary 4.6. Let $X$ be a Banach space. A metric component $\mathcal{H}$ of $\operatorname{Conv}_{\mathrm{H}}(X)$ is homeomorphic to an infinitedimensional Hilbert space if and only if $\mathcal{H}$ is not locally compact.

This corollary reduces the problem of recognition of the topology of non-locally compact components of $\operatorname{Conv}_{\mathrm{H}}(X)$ to calculating their densities. This problem was considered in [3] and [4). In particular, 3] contains the following characterization:

Proposition 4.7. For a Banach space $X$ and a metric component $\mathcal{H}$ of the space $\mathrm{Conv}_{\mathrm{H}}(X)$ the following conditions are equivalent:

(1) $\mathcal{H}$ is separable;

(2) $\operatorname{dens}(\mathcal{H})<\mathfrak{c}$;

(3) $\mathcal{H}$ contains a polyhedral convex set;

(4) the characteristic cone $V_{\mathcal{H}}$ is polyhedral and belongs to $\mathcal{H}$;

Remark 4.8. Each metric component of $\operatorname{Conv}_{\mathrm{H}}(X)$ being homeomorphic to a convex set, is connected and thus coincides with a connected component of $\operatorname{Conv}_{\mathrm{H}}(X)$. Hence there is no difference between metric and connected components of $\operatorname{Conv}_{\mathrm{H}}(X)$, so using the term component of Conv $(X)$ (without an adjective "metric" or "connected") will not lead to misunderstanding.

\section{Operators Between spaces of CONVEX Sets}

Each linear continuous operator $T: X \rightarrow Y$ between normed spaces induces a map $\bar{T}: \operatorname{Conv}_{\mathrm{H}}(X) \rightarrow \operatorname{Conv}_{\mathrm{H}}(Y)$ assigning to each closed convex set $A \in \operatorname{Conv}_{\mathrm{H}}(X)$ the closure $\overline{T(A)}$ of its image $T(A)$ in $Y$. In this section we study properties of the induced operator $\bar{T}$. We start with algebraic properties that trivially follow from the linearity and continuity of the operator $T$.

Proposition 5.1. If $T: X \rightarrow Y$ is a linear continuous operator between Banach spaces and $\bar{T}: \operatorname{ConvH}_{\mathrm{H}}(X) \rightarrow$ $\operatorname{Conv}_{\mathrm{H}}(X)$ is the induced operator, then 
(1) $\bar{T}(\max \{A, B\})=\max \{\bar{T}(A), \bar{T}(B)\}$;

(2) $\bar{T}(r \cdot A)=r \cdot \bar{T}(A)$;

(3) $\bar{T}(A \oplus B)=\bar{T}(A) \oplus \overline{T(B)}$;

(4) $\bar{T}((1-t) A \oplus t B)=(1-t) \bar{T}(A) \oplus t \overline{T(B)}$;

for any sets $A, B \in \operatorname{Conv}_{\mathbf{H}}(X)$ and real numbers $r \in \mathbb{R}$ and $t \in[0,1]$.

We shall be mainly interested in the operators $\bar{T}$ induced by quotient operators $T$. We recall that for a closed linear subspace $Z$ of a normed space $X$ the quotient normed space $X / Z=\{x+Z: x \in X\}$ carries the quotient norm

$$
\|x+Z\|=\inf _{y \in x+Z}\|y\| .
$$

By $q: X \rightarrow X / Z, q: x \mapsto x+Z$ we shall denote the quotient operator and by $\bar{q}: \operatorname{Conv}_{\mathrm{H}}(X) \rightarrow \operatorname{Conv}_{\mathrm{H}}(X / Z)$ the induced operator between the spaces of closed convex sets.

For a closed convex set $C \subset X$ by $C / Z$ we denote the image $q(C) \subset X / Z$. So, $\bar{q}(C)=\overline{C / Z}$. If $Z \subset V_{C}$, then the set $C / Z$ is closed in $X / Z$ and hence $\bar{q}(C)=C / Z$. Indeed, $Z \subset V_{C}$ implies that $C+Z=C$ and hence $C / Z=(X / Z) \backslash q(X \backslash C)$ is closed in $X / Z$ being the complement of the set $q(X / \backslash C)$ which is open as the image of the open set $X \backslash C$ under the open map $q: X \rightarrow X / Z$.

We shall need the following simple reduction lemma:

Lemma 5.2. Let $Z$ be a closed linear subspace of a normed space $X$ and $A, B$ are non-empty closed convex subsets of $X$. If $Z \subset V_{A} \cap V_{B}$, then $\mathrm{d}_{\mathrm{H}}(A, B)=\mathrm{d}_{\mathrm{H}}(A / Z, B / Z)$.

Proof. The inequality $\mathrm{d}_{\mathrm{H}}(A / Z, B / Z) \leq \mathrm{d}_{\mathrm{H}}(A, B)$ follows from $\|q\| \leq 1$. Assuming that $\mathrm{d}_{\mathrm{H}}(A / Z, B / Z)<\mathrm{d}_{\mathrm{H}}(A, B)$, we can find a point $a \in A$ with $\operatorname{dist}(a, B)>\mathrm{d}_{\mathrm{H}}(A / Z, b / Z)$ or a point $b \in B$ with $\operatorname{dist}(b, A)>\mathrm{d}_{\mathrm{H}}(A / Z, B / Z)$. Without loss of generality, we can assume that $\operatorname{dist}(a, B)>\mathrm{d}_{\mathrm{H}}(A / Z, B / Z)$ for some point $a \in A$. Consider its image $a^{\prime}=q(a) \in A / Z$ under the quotient operator $q: X \rightarrow X / Z$. By the definition of the Hausdorff metric, $\mathrm{d}_{\mathrm{H}}(A / Z, B / Z)<\operatorname{dist}(a, B)$, there is a point $b^{\prime} \in B / Z$ such that $\left\|b^{\prime}-a^{\prime}\right\|<\operatorname{dist}(a, B)$. By the definition of the quotient norm, there is a vector $z \in Z$ such that $q(z)=b^{\prime}-a^{\prime}$ and $\|z\|<\operatorname{dist}(a, B)$. Now consider the point $b=a+z$ and observe that $q(b)=q(a)+q(z)=a^{\prime}+b^{\prime}-a^{\prime}=b^{\prime} \in B / Z$ and hence $b \in q^{-1}(B / Z)=B+Z=B$. So, $\operatorname{dist}(a, B) \leq\|a-b\|=\|z\|<\operatorname{dist}(a, B)$, which is a desired contradiction that completes the proof of the equality $\mathrm{d}_{\mathrm{H}}(A, B)=\mathrm{d}_{\mathrm{H}}(A / Z, B / Z)$.

Corollary 5.3. Let $X$ be a normed space $X, \mathcal{H}$ be a component of the space $\operatorname{Conv}_{\mathrm{H}}(X)$, and $Z$ be a closed linear subspace of $X$. If $Z \subset V_{\mathcal{H}}$, then the quotient operator

$$
\bar{q}: \mathcal{H} \rightarrow \mathcal{H} / Z, \quad \bar{q}: C \mapsto C / Z,
$$

maps isometrically the component $\mathcal{H}$ of $\operatorname{Conv}_{\mathrm{H}}(X)$ onto the component $\mathcal{H} / Z$ of $\operatorname{Conv}_{\mathrm{H}}(X / Z)$ containing some (equivalently, each) convex set $C / Z$ with $C \in \mathcal{H}$.

\section{Proof of Theorem 1}

Let $X$ be a Banach space and $\mathcal{H}$ be a component of the space $\operatorname{Conv}_{\mathrm{H}}(X)$.

If $\mathcal{H}$ contains no polyhedral convex set, then by Propositions 4.7, it has density $\operatorname{dens}(\mathcal{H}) \geq \mathfrak{c}$. Consequently, $\mathcal{H}$ is not locally compact and by Corollary 4.6. $\mathcal{H}$ is homeomorphic to the non-separable Hilbert space $l_{2}(\kappa)$ of density $\kappa=\operatorname{dens}(\mathcal{H}) \geq \mathfrak{c}$.

It remains to analyze the topological structure of $\mathcal{H}$ if it contains a polyhedral convex set. In this case Proposition 4.7 guarantees that the characteristic cone $V_{\mathcal{H}}$ belongs to $\mathcal{H}$ and is polyhedral in $X$. If $V_{\mathcal{H}}=X$, then $\mathcal{H}=\{X\}$ is a singleton. So, we assume that $V_{\mathcal{H}} \neq X$. Since the cone $V_{\mathcal{H}}$ is polyhedral, the the closed linear subspace $Z=-V_{\mathcal{H}} \cap V_{\mathcal{H}}$ has finite codimension in $X$. Then the quotient Banach space $\tilde{X}=X / Z$ is finite-dimensional. Let $q: X \rightarrow \tilde{X}$ be the quotient operator.

By Corollary 5.3, the component $\mathcal{H}$ is isometric to the component $\tilde{\mathcal{H}}=\mathcal{H} / Z$ of the space $\operatorname{Conv}_{\mathrm{H}}(\tilde{X})$ of closed convex subsets of the finite-dimensional Banach space $\tilde{X}$. The component $\tilde{\mathcal{H}}$ contains the polyhedral convex cone $V_{\tilde{\mathcal{H}}}=q\left(V_{\mathcal{H}}\right)$ which has the property $-V_{\tilde{\mathcal{H}}} \cap V_{\tilde{\mathcal{H}}}=\{0\}$.

The cone $V_{\tilde{\mathcal{H}}}$ can be of two types.

1. The cone $V_{\tilde{\mathcal{H}}}=\{0\}$ is trivial. In this case $\mathcal{H}$ contains the closed linear subspace $Z=V_{\mathcal{H}}$ of finite codimension in $X$. Taking into account that $V_{\mathcal{H}} \neq X$, we conclude that $\operatorname{dim}(\tilde{X}) \geq 1$. Depending on the value of $\operatorname{dim}(\tilde{X})$, we have two subcases.

1a. The dimension $\operatorname{dim}(\tilde{X})=1$ and hence $\mathcal{H}$ contains the linear subspace $Z=V_{\mathcal{H}}$ of codimension 1 in $X$. In this case $\tilde{\mathcal{H}}$ coincides with the space $\operatorname{BConv}_{H}(\tilde{X})$ of non-empty bounded closed convex subsets of the one-dimensional Banach space $\tilde{X}$ and hence $\tilde{\mathcal{H}}$ is isometric to the half-plane $\mathbb{R} \times \overline{\mathbb{R}}_{+}$. 
1b. The dimension $\operatorname{dim}(\tilde{X}) \geq 2$ and hence $\mathcal{H}$ contains the linear subspace $Z$ of codimension $\geq 2$ in $X$. In this case $\tilde{\mathcal{H}}$ coincides with the space $\operatorname{BConv}_{H}(\tilde{X})$ of non-empty bounded closed convex subsets of the finite-dimensional Banach space $\tilde{X}$ of finite dimension $\operatorname{dim}(\tilde{X}) \geq 2$. By the result of Nadler, Quinn and Stavrakas 9 , the space $\operatorname{BConv}_{H}(\tilde{X})$ is homeomorphic to the Hilbert cube manifold $Q \times \overline{\mathbb{R}}_{+}$.

2. The characteristic cone $V_{\tilde{\mathcal{H}}} \neq\{0\}$ is not trivial. This case has two subcases.

2a. $\operatorname{dim}(\tilde{X})=\operatorname{dim}\left(V_{\tilde{\mathcal{H}}}\right)=1$. In this case the component $\mathcal{H}$ (and its isometric copy $\mathcal{H}$ ) is isometric to the real line $\mathbb{R}$.

2b. $\operatorname{dim}(\tilde{X}) \geq 2$. In this case we shall prove that the component $\tilde{\mathcal{H}}$ (and its isometric copy $\mathcal{H}$ ) is homeomorphic to the separable Hilbert space $l_{2}$. This will follow from the separability of $\mathcal{H}$ and Corollary 4.6 as soon as we check that the space $\tilde{\mathcal{H}}$ is not locally compact. To prove this fact, it suffices for every positive $\varepsilon<1$ to construct a sequence of closed convex sets $\left\{C_{n}\right\}_{n \in \mathbb{N}} \subset \tilde{\mathcal{H}}$ such that $\mathrm{d}_{\mathrm{H}}\left(C_{n}, V_{\tilde{\mathcal{H}}}\right) \leq \varepsilon$ and $\inf _{n \neq m} \mathrm{~d}_{\mathrm{H}}\left(C_{n}, C_{m}\right)>0$.

The cone $V_{\tilde{\mathcal{H}}}$ is polyhedral and hence is generated by for some finite set $E \subset \tilde{X} \backslash\{0\}$, see [8] or Theorem 1.1 of [17. For every $e \in E$ the vector $-e$ does not belong to $V_{\tilde{\mathcal{H}}}$. Then the Hahn-Banach Theorem yields a linear functional $h_{e} \in X^{*}$ such that $h_{e}(-e)<\inf h_{e}\left(V_{\tilde{\mathcal{H}}}\right)=0$. It can be shown that the functional $h=\sum_{e \in E} h_{e}$ has the property $h(v)>0$ for all $v \in V_{\tilde{\mathcal{H}}} \backslash\{0\}$.

Since $\operatorname{dim}(\tilde{X}) \geq 2$ and $V_{\tilde{\mathcal{H}}} \neq \tilde{X}$, we can find a non-zero linear continuous functional $f: \tilde{X} \rightarrow \mathbb{R}$ such that $\sup f\left(V_{\tilde{\mathcal{H}}}\right)=0$ and the intersection $f^{-1}(0) \cap V_{\tilde{\mathcal{H}}}$ contains a non-zero vector $x \in \tilde{X}$. Multiplying $x$ by a suitable positive constant, we can assume that $h(x)=1$. Since $h^{-1}(0) \cap V_{\tilde{\mathcal{H}}}=\{0\} \neq f^{-1}(0) \cap V_{\tilde{\mathcal{H}}}$, the functionals $h$ and $f$ are distinct and hence there is a vector $y \in h^{-1}(0) \backslash f^{-1}(0)$ with norm $\|y\|=\varepsilon$. Replacing $y$ by $-y$, if necessary, we can assume that $f(y)>0$.

For every $n \in \omega$ consider the point $c_{n}=3^{n} x+y$ and the closed convex set

$$
C_{n}=\max \left\{V_{\tilde{\mathcal{H}}},\left\{c_{n}\right\}\right\}=\overline{\operatorname{conv}}\left(V_{\tilde{\mathcal{H}}} \cup\left\{c_{n}\right\}\right) \subset \tilde{X} .
$$

It follows from $x \in V_{\tilde{\mathcal{H}}}$ and $\operatorname{dist}\left(c_{n}, V_{\tilde{\mathcal{H}}}\right) \leq \operatorname{dist}\left(3^{n} x+y, 3^{n} x\right)=\|y\|=\varepsilon$ that $\mathrm{d}_{\mathrm{H}}\left(C_{n}, V_{\tilde{\mathcal{H}}}\right) \leq \varepsilon$.

We claim that $\inf _{n \neq m} \mathrm{~d}_{\mathrm{H}}\left(C_{n}, C_{m}\right) \geq \delta$ where

$$
\delta=\frac{1}{2} f(y) \leq \frac{1}{2}\|y\|=\frac{1}{2} \varepsilon<\frac{1}{2} .
$$

This will follow as soon as we check that $\operatorname{dist}\left(c_{n}, C_{m}\right) \geq \delta$ for any numbers $n<m$.

Assuming conversely that $\operatorname{dist}\left(c_{n}, C_{m}\right)<\delta$ and taking into account that the convex $\operatorname{set} \operatorname{conv}\left(V_{\tilde{\mathcal{H}}} \cup\left\{c_{m}\right\}\right)$ is dense in $C_{m}$, we can find a point $c \in \operatorname{conv}\left(V_{\tilde{\mathcal{H}}} \cup\left\{c_{m}\right\}\right)$ such that $\operatorname{dist}\left(c_{n}, c\right)<\delta$. The point $c$ belongs to the convex hull of the set $V_{\tilde{\mathcal{H}}} \cup\left\{c_{m}\right\}$ and hence can be written as a convex combination $c=t c_{m}+(1-t) v=t\left(3^{m} x+y\right)+(1-t) v$ for some $t \in[0,1]$ and $v \in V_{\tilde{\mathcal{H}}}$. Observe that $h\left(c_{n}\right)=h\left(3^{n} x+y\right)=3^{n} h(x)+h(y)=3^{m} \cdot 1+0=3^{n}$ while $h(c)=\operatorname{th}\left(c_{m}\right)+(1-t) h(v) \geq t h\left(c_{m}\right)=3^{m} t$. Then

$$
3^{m} t-3^{n} \leq h(c)-h\left(c_{n}\right) \leq\left|h(c)-h\left(c_{n}\right)\right| \leq\|h\| \cdot\left\|c-c_{n}\right\|<1 \cdot \delta
$$

and hence

$$
t<3^{n-m}+3^{-m} \delta \leq \frac{1}{3}+\frac{1}{3} \delta<\frac{1}{3}+\frac{1}{6}=\frac{1}{2} .
$$

Next, we apply the functional $f$ to the points $c_{n}$ and $c$. Since $f(x)=0$, we get $f\left(c_{n}\right)=f\left(3^{n} x+y\right)=f(y)=2 \delta$. On the other hand, $f\left(V_{\tilde{\mathcal{H}}}\right) \subset(-\infty, 0]$ implies $f(v) \leq 0$ and hence

$$
f(c)=f\left(t c_{m}+(1-t) v\right)=t f\left(3^{m} x+y\right)+(1-t) f(v)=t f(y)+(1-t) f(v) \leq t f(y)=2 \delta t .
$$

Then

$$
\delta=2 \delta\left(1-\frac{1}{2}\right)<2 \delta(1-t) \leq\left|f\left(c_{n}\right)-f(c)\right| \leq\|f\| \cdot\left\|c_{n}-c\right\|<\delta,
$$

which is a desired contradiction.

The above proof can be visualized in the following picture:

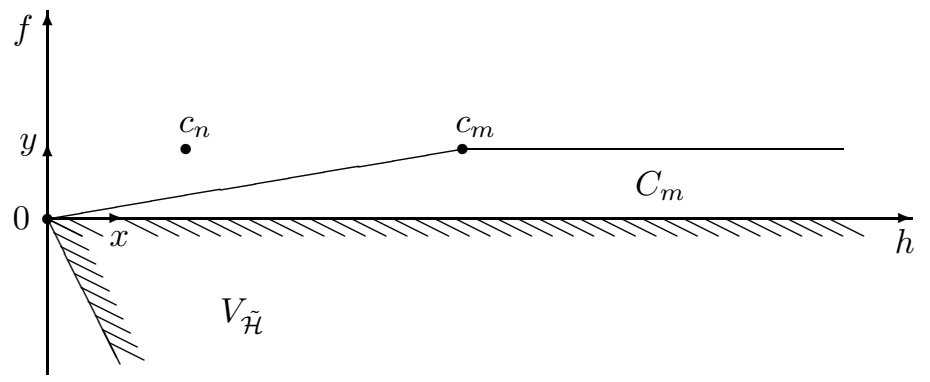




\section{REFERENCES}

[1] T. Banakh, R. Cauty, Topological classification of convex sets in Frechet spaces, Studia Math. 205 (2011), 1-11.

[2] T. Banakh, I. Hetman, A "hidden" characterization of polyhedral convex sets, Studia Math. 206 (2011), 63-74.

[3] T. Banakh, I. Hetman, A "hidden" characterization of approximatively polyhedral convex sets in Banach spaces, preprint (http://arxiv.org/abs/1111.6708).

[4] T. Banakh, I. Hetman, The hiding and polyhedrality numbers of closed convex sets in Banach spaces, in preparation.

[5] A.S. Granero, M.S. Jimenez, J.P. Moreno, Convex sets in Banach spaces and a problem of Rolewicz, Studia Math. 129:1 (1998), $19-29$.

[6] A. Granero, M. Jimenez, A. Montesinos, J. Moreno, A. Plichko, On the Kunen-Shelah properties in Banach spaces, Studia Math. 157:2 (2003), 97-120.

[7] P. Hájek, S. Montesinos, J. Vanderwerff, V. Zizler, Biorthogonal systems in Banach spaces, Springer, New York, 2008.

[8] V. Klee, Some characterizations of convex polyhedra, Acta Math. 102 (1959) 79-107.

[9] S. Nadler, J. Quinn, N.M. Stavrakas, Hyperspaces of compact convex sets, Pacific J. Math. 83 (1979), 441-462.

[10] S. Negrepontis, Banach Spaces and Topology, in: Handbook of Set-Theoretic Topology, North-Holland, 1984, p. $1045-1142$.

[11] A. Plichko, Existence of a bounded total biorthogonal system in a Banach space, Teor. Funks. Funkt. Anal. Pril. 33 (1980), 111-118.

[12] K. Sakai, The spaces of compact convex sets and bounded closed convex sets in a Banach space, Houston J. Math. 34:1 (2008), 289-300.

[13] S. Shelah, Uncountable constructions for B.A., e.c. groups and Banach spaces, Israel J. Math. 51:4 (1985), $273-297$.

[14] S. Todorcevic, Biorthogonal systems and quotient spaces via Baire category methods, Math. Ann. 335:3 (2006), $687-715$.

[15] H.Toruńczyk, Characterizing Hilbert space topology, Fund. Math. 111:3 (1981), 247-262.

[16] H. Torunczyk, A correction of two papers concerning Hilbert manifolds, Fund. Math. 125:1 (1985), 89-93.

[17] G. Ziegler, Lectures on polytopes, Springer-Verlag, New York, 1995.

(T. Banakh) Universytet Jana Kochanowskiego, Kielce (Poland) and Ivan Franko National University of Lviv (Ukraine) E-mail address: tbanakh@franko.lviv.ua

(I.Getman) Department of Mathematics, Ivan Franko National University of Lviv, Universytetska 1, Lviv, 79000, UKRAINE

E-mail address: ihromant@gmail.com

(K. Sakai) Institute of Mathematics, University of Tsukuba, Tsukuba, 305-8571, Japan

E-mail address: sakaiktr@sakura.cc.tsukuba.ac.jp 